\title{
The dyslipidemia-associated SNP on the APOA1/ C3/A5 gene cluster predicts post-surgery poor outcome in Taiwanese breast cancer patients: a 10-year follow-up study
}

Mei-Chi Hsu ${ }^{1,2}$, Kuo-Ting Lee ${ }^{3}$, Wei-Chiang Hsiao ${ }^{4}$, Chih-Hsing Wu ${ }^{5}$, Hung-Yu Sun², I-Ling Lin ${ }^{6 \dagger}$ and Kung-Chia Young ${ }^{1,2,7^{*}+}$

\begin{abstract}
Background: Post-surgery therapies are given to early-stage breast cancer patients due to the possibility of residual micrometastasis, and optimized by clincopathological parameters such as tumor stage, and hormone receptor/ lymph node status. However, current efficacy of post-surgery therapies is unsatisfactory, and may be varied according to unidentified patient genetic factors. Increases of breast cancer occurrence and recurrence have been associated with dyslipidemia, which can attribute to other known risk factors of breast cancer including obesity, diabetes and metabolic syndrome. Thus we reasoned that dyslipidemia-associated nucleotide polymorphisms (SNPS) on the APOA1/C3/A5 gene cluster may predict breast cancer risk and tumor progression.

Methods: We analyzed the distribution of 5 selected APOA1/C3/A5 SNPs in recruited Taiwanese breast cancer patients ( $n=223$ ) and healthy controls ( $n=162$ ). The association of SNP (APOA1 rs670) showing correlation with breast cancer with baseline and follow-up parameters was further examined.

Results: APOA1 rs670 A allele carriage was higher in breast cancer patients than controls (59.64\% vs. 48.77\%, $\mathrm{p}=0.038$ ). The rs670 A allele carrying patients showed less favorable baseline phenotype with positive lymph nodes $(\mathrm{G} / \mathrm{A}: \mathrm{OR}=3.32,95 \% \mathrm{Cl}=1.77-6.20, \mathrm{p}<0.001 ; \mathrm{A} / \mathrm{A}: \mathrm{OR}=2.58,95 \% \mathrm{Cl}=1.05-6.32, \mathrm{p}=0.039)$ and negative hormone receptor expression ( $A / A$ : $O R=4.85,95 \% \mathrm{Cl}=1.83-12.83, \mathrm{p}=0.001$ ) in comparison to $\mathrm{G} / \mathrm{G}$ carriers. Moreover, $\mathrm{r} 670 \mathrm{~A} / \mathrm{A}$ carrying patients had higher risks in both tumor recurrence $(H R=3.12,95 \% \mathrm{Cl}=1.29-7.56, p=0.012)$ and mortality $(H R=4.36$, 95\% $\mathrm{Cl}=1.52-12.47, \mathrm{p}=0.006)$ than patients with no A alleles after adjustments for associated baseline parameters. Furthermore, the prognostic effect of rs670 A/A carriage was most evident in lymph node-negative patients, conferring to the highest risks of recurrence ( $H R=4.98,95 \% \mathrm{Cl}=1.40-17.70, \mathrm{p}=0.013)$ and mortality $(\mathrm{HR}=9.87,95 \%$ $\mathrm{Cl}=1.60-60.81, \mathrm{p}=0.014)$ than patients with no A alleles.

(Continued on next page)
\end{abstract}

\footnotetext{
* Correspondence: t7908077@mail.ncku.edu.tw

${ }^{\dagger}$ Equal contributors

'Research Center for Medical Laboratory Biotechnology, College of Medicine,

National Cheng Kung University, Tainan, Taiwan

2Department of Medical Laboratory Science and Biotechnology, College of

Medicine, National Cheng Kung University, Tainan, Taiwan

Full list of author information is available at the end of the article
} 
(Continued from previous page)

Conclusions: APOA1 rs670 A/A carriage showed poor post-surgery prognosis in Taiwanese lymph node-negative breast cancer patients, whose prognosis were considered better and adjuvant treatment might be less stringent according to currently available assessment protocols. Our findings suggest that APOA1 rs670 indicate a post-surgery risk of breast cancer disease progression, and that carriers of this SNP may benefit from more advanced disease monitoring and therapy regimens than the current regular standards. Furthermore, control of lipid homeostasis might protect APOA1 rs670 minor allele carriers from breast cancer occurrence and progression.

Keywords: APOA1/C3/A5, SNP, APOA1 rs670, Lymph node micrometastasis, Post-surgery, Prognosis

\section{Background}

Breast cancer is currently the leading cause of cancer deaths in females worldwide, and also the second most common cancer after lung cancer [1]. The current therapeutic regimens for operable early-stage breast cancers include endocrine therapy, chemotherapy and radiotherapy. The assessment tools for post-surgery planning are currently based on clinicopathological evaluations, but are unsatisfactory requiring finer adjustments [2]. The risk factors for breast cancer poor prognosis include positive sentinel lymph node metastasis, hormone receptor negativity, larger tumor size, younger age, and menopausal status [2]. In contrast to the Western population, the breast cancer incidence peaks at a younger age in Oriental Asians, which include Taiwanese [3,4]. Nevertheless, the westernized dietary pattern and lifestyle in Taiwan in the last two decades has increased the incidences of metabolic disorders, including dyslipidemia, as well as breast cancer in Taiwanese females [4].

Both cohort and case-control epidemiological observations have demonstrated the association of metabolic syndrome, obesity and diabetes with increased breast cancer risk [5,6]. Dyslipidemia often occur in parallel to obesity and diabetes, and is a component of metabolic syndrome [7]. Dyslipidemia in the forms of hypertriglyceridemia, hypercholesterolemia, and low high density lipoprotein-cholesterol (HDL-C) have been observed in breast cancer patients of several ethnic groups [8-11]. Moreover, in large cohort studies dyslipidemia is associated with increased breast cancer risk and poor prognosis [12-14]. The intake of lipid-lowering drugs or agents in women was associated with reduced breast cancer occurrence and recurrence risk [15-17]. Consistently, mammary tumor growth and metastasis was accelerated in a hyperlipidemic murine model [18].

The $A P O A 1 / C 3 / A 5$ gene cluster transcribes for apolipoproteins (apo) A1, C3 and A5, which regulate HDL-formation and lipoprotein lipase activity [19]. Singlenucleotide polymorphisms (SNPs) on the APOA1/C3/A5 gene cluster are associated with metabolic syndrome, dyslipidemia and diabetes [19]. APOA1 rs670 is associated with altered $\mathrm{HDL}-\mathrm{C}$ levels, and increased risks of coronary artery disease and metabolic syndrome [20-25]. APOC3 rs2854116 and rs2854117 are correlated to insulin resistance and non-alcoholic liver steatosis [26]. APOA5 rs662799 and rs2075291 carriers are known to have increased plasma triglyceride [27,28], and our recent study showed correlation of APOA5 rs662799 with central obesity in males [29]. Though the effects of these SNPs on APOA1/C3/A5 on metabolic disorders have been widely studied and reviewed, their contributions to breast cancer have not been determined in detail.

In this study, we first tested the correlation of 5 selected well-known SNPs on the APOA1/C3/A5 gene cluster with breast cancer in a case-control manner. Furthermore, we analyzed the effect of the SNPs associated with greatest breast cancer risk on patient baseline tumor characteristics, and their post-surgery outcome after a mean follow-up period of 10.4 years. Finally, we specifically examined the prognostic value of APOA1 rs670 in lymph node-negative breast cancer patients.

\section{Methods}

\section{Patient recruitment}

Taiwanese female breast cancer patients $(n=223,48.4 \pm$ 10.2 years, ranged 29-75 years) received surgical intervention plus axillary/sentinel lymph node dissection during 1999-2005 at National Cheng Kung University Hospital (NCKUH) and Tainan Hospital, and were followed-up to November 2012. Healthy female controls $(\mathrm{n}=162,43.0 \pm 8.8$ years, ranged $19.0-69.0$ years.) were also recruited. This is a continuation study of Hsiao et al., 2004 [30]. Bodyweight ( $\mathrm{kg}$ ) and body height (m) were measured at the time of mastectomy, and used for calculating body mass index (BMI, $\mathrm{kg} \mathrm{m}^{-2}$ ). The diagnosis was confirmed by histological examinations of mammary and node specimens. Estrogen receptor (ER) and progesteron receptor (PR) expressions in primary breast tumor were determined as described previously [31]. Individuals with at least one first-degree or second-degree female relatives affected by breast cancer were considered to have a family history. This information was obtained by interview with patients and their family members. The recruited patients received tamoxifen (TAM) $(n=92)$, TAM and chemotherapy $(n=56)$, TAM and radiotherapy $(n=10)$, and triple-therapy $(n=55)$. This 
study received approval from the local institutional review board (NCKUH IRB) and signed informed consent was obtained from the patients.

\section{Genomic DNA extraction, SNP genotyping and linkage analysis}

Genomic DNA was extracted from white blood cells using the Puregene DNA Isolation Kit (Gentra Systems, Minneapolis, MN, USA). According to criteria described in Hsu et al. [29], we selected five SNPs on the APOA1/ $C 3 / A 4 / A 5$ gene cluster that follows: $A P O A 1$ rs670, APOC3 rs2854116, APOC3 rs2854117, APOA5 rs662799 and $A P O A 5$ rs2075291. The SNP genotypes were determined using commercial real-time PCR primer and probes from Applied Biosystems (ABI, Foster City, CA, USA) (APOA5 rs662799 and APOA5 rs2075291) and TIB MOBIOL (Berlin, Germany) (APOA1 rs670, APOC3 rs2854116 and $A P O C 3$ rs2854117). Fluorescence data from real-time PCRs were collected by a Step-One-Plus Sequence Detection System (ABI) or LightCycler 480 (Roche, St. Louis, MO, USA). Haploview [32] was used for the analysis of SNP linkage disequilibrium, and Hardy-Weinberg equilibrium, and haplotype analysis. SNP linkage disequilibrium test results with logarithm of odds (LOD) socores $\geq 2$ and pair-wise D'>0.80 were considered as significant linkage.

\section{Statistical analysis}

The association of SNP with breast cancer risk, baseline clinical parameters and post-surgery progression was analyzed by Chi-squared test. The odds ratio for unfavorable baseline characteristics and events in post-surgery progression was analyzed by binary or multi-nominal logistic analysis. The differences in BMI, age, and mean years in survival were analyzed by one-way ANOVA. The survival curves of $A P O A 1$ rs670 genotype carriers were plotted by Kaplan-Meier analysis. The hazard ratio for overall and recurrence-free survival was calculated by Cox proportional-hazards regression analysis. Possible confounders including unfavorable baseline characteristics, age and BMI were adjusted for in regression analysis. Statistical analysis was performed using SPSS 13 (SPSS Inc., Chicago, DE, USA). In all cases, p-values $\leq 0.05$ were considered statistically significant.

\section{Results}

\section{Demographic characteristics of recruited breast} cancer patients

The baseline characteristics of the recruited breast cancer patients are shown in Table 1. The mean BMI of breast cancer patients at baseline was $23.36 \pm 3.79 \mathrm{~kg} \mathrm{~m}^{-2}$. The major tumor type of recruited patients was infiltrating ductal carcinoma (86.55\%), and the tumor occurrence side was evenly distributed (right breast: $44.84 \%$,
Table 1 Demographic data of recruited breast cancer patients $(n=223)$

\begin{tabular}{|c|c|}
\hline Parameters & Values \\
\hline \multicolumn{2}{|l|}{ Baseline } \\
\hline Age (years) & $48.4 \pm 10.2(29-75)$ \\
\hline $\mathrm{BMI}^{\mathrm{a}}\left(\mathrm{kg} \mathrm{m}^{-2}\right)$ & $23.36 \pm 3.79(15.06-38.05)$ \\
\hline \multicolumn{2}{|l|}{ Tumor type } \\
\hline Infiltrating ductal carcinoma & $193(86.55 \%)$ \\
\hline Ductal carcinoma in situ & $17(7.62 \%)$ \\
\hline Others & $11(4.93 \%)$ \\
\hline Unknown & $2(0.90 \%)$ \\
\hline \multicolumn{2}{|l|}{ Side } \\
\hline Right & $100(44.84 \%)$ \\
\hline Left & $114(51.12 \%)$ \\
\hline Both & $9(4.04 \%)$ \\
\hline \multicolumn{2}{|l|}{ Tumor size } \\
\hline$<5 \mathrm{~cm}$ & $159(71.30 \%)$ \\
\hline$\geq 5 \mathrm{~cm}$ & $26(11.66 \%)$ \\
\hline Unknown & $38(17.04 \%)$ \\
\hline \multicolumn{2}{|l|}{ Lymph node involvement } \\
\hline Positive & $121(53.81 \%)$ \\
\hline Negative & $90(40.36 \%)$ \\
\hline Unknown & $12(5.83 \%)$ \\
\hline \multicolumn{2}{|l|}{ TMN staging } \\
\hline Stages 0-2 & $154(69.06 \%)$ \\
\hline Stages 3-4 & $30(13.45 \%)$ \\
\hline Unknown & 39 (17.49\%) \\
\hline \multicolumn{2}{|l|}{ ER/PR status } \\
\hline Single or double positive & $144(64.57 \%)$ \\
\hline Negative & $52(23.32 \%)$ \\
\hline Unknown & $27(12.11 \%)$ \\
\hline \multicolumn{2}{|l|}{ Family history } \\
\hline Positive & $13(5.83 \%)$ \\
\hline Negative & $203(91.03 \%)$ \\
\hline Unknown & $7(3.14 \%)$ \\
\hline \multicolumn{2}{|l|}{ Post-surgery follow-up } \\
\hline \multicolumn{2}{|l|}{ Therapies } \\
\hline TAM only & $92(41.26 \%)$ \\
\hline TAM and chemotherapy & $56(25.11 \%)$ \\
\hline TAM and radiotherapy & $10(4.48 \%)$ \\
\hline Triple therapy & 55 (24.66\%) \\
\hline Unknown & $10(4.48 \%)$ \\
\hline \multicolumn{2}{|l|}{ Recurrence } \\
\hline With & $56(25.11 \%)$ \\
\hline Without & 167 (74.89\%) \\
\hline
\end{tabular}

Mortality 
Table 1 Demographic data of recruited breast cancer patients ( $n=223$ ) (Continued)

\begin{tabular}{lc}
\hline With & $33(14.80 \%)$ \\
Without & $190(85.20 \%)$ \\
Progression-free & $165(73.99 \%)$ \\
Mean survival years & \\
Recurrence-free & $9.26 \pm 5.31(0.04-24.52)$ \\
Overall & $10.14 \pm 5.11(0.14-24.52)$ \\
\hline $\begin{array}{l}\text { NOTE: Values shown are mean } \pm \text { s.d., or number of patients }(\% \text { of } n) . \\
\text { aData from 185 patients. Bold type indicates } p<0.050 .\end{array}$ \\
$\begin{array}{l}\text { Abbreviations: BMI Body mass index, } E R \text { estrogen receptor, } P R \text { progesterone } \\
\text { receptor, TAM tamoxifen. }\end{array}$
\end{tabular}

left breast: $51.12 \%$, bilateral: $4.04 \%)$. The recruited patients were mainly of early-stage breast cancer, as $71.30 \%$ of patients had tumors $<5 \mathrm{~cm}, 69.06 \%$ were of tumor stages $0-2$, and $64.57 \%$ of patients had single or double positive for ER and PR. Additionally, 53.81\% of patients had detectable lymph node involvement, and only $5.83 \%$ had a family history of breast cancer. The follow-up period of the breast cancer patients ranged from 0.14 to 24.52 years (median: 9.93 years), mounting to 2261.68 person-years in total. About three-forth of patients (73.99\%) remained progression-free of recurrence or death. The mean years for recurrence-free and overall survival were $9.26 \pm 5.31$ and $10.14 \pm 5.11$, respectively (Table 1).

\section{ApoA1 rs670 was associated with increased breast cancer risk}

The distributions of the tested SNPs (APOA1 rs670, APOC3 rs2854116, APOC3 rs2854117, APOA5 rs662799 and $A P O A 5$ rs2075291) fitted the Hardy-Weinberg equilibrium in both breast cancer patients and healthy controls (Additional file 1). Different linkage patterns of the tested SNPs were observed between breast cancer patients (Additional file 2). Significant linkage (LOD $\geq 2$, D'> 0.80) was observed among APOA1 rs670, APOC3 rs2854116 and $A P O C 3$ rs2854117 $\left(D^{\prime}=0.84-0.88\right)$, and between $A P O A 5$ rs662799 and APOA5 rs2075291 in healthy controls $\left(D^{\prime}=0.86\right)$. In contrast, the linkage among $A P O A 1$ rs670, APOC3 rs2854116 and APOC3 rs2854117 was lost in breast cancer patients, while significant linkage was only observed between the two $A P O A 5$ SNPs $\left(\mathrm{D}^{\prime}=1.00\right)$.

The frequency of $A P O A 1 \mathrm{rs} 670$ A allele (G/A + A/A) carriers in breast cancer patients was significantly higher than that in healthy controls $(59.64 \%$ vs. $48.77 \%, p=0.038$, Additional file 3). On the other hand, the genotype and allele frequencies of all other SNPs were comparable between breast cancer patients and healthy controls. The post-surgery therapies received by the different APOA1 rs670 genotype carriers were comparable $(p=0.151)$. Therefore we tested the contribution of APOA1 rs670 to breast cancer in recruited patients cross-sectionally at baseline and longitudinally at follow-up.

\section{APOA1 rs670 was associated with sentinel lymph node-positivity and tumor ER/PR negativity at baseline} Upon analysis of APOA1 rs670 against breast tumor clinical parameters, we found the mean age and BMI of different APOA1 rs670 genotype carriers were comparable at baseline (Table 2). In contrast, APOA1 rs670 was associated with nodal involvement as well as tumor hormone receptor expression (Table 2 and 3). Baseline lymph node positivity was more frequent in patients carrying APOA1 rs670 G/A or A/A genotypes than in those carrying G/G genotype (G/A: $51.89 \%$, A/A: $48.15 \%$, G/G: 24.44\%, $p=0.002$ ) (Table 2). Moreover, APOA1 rs670 G/ A or A/A carriage also significantly increased the frequency of having tumors double negative for ER/PR expression than G/G carriage in patients (G/A: 24.53\%, A/ A: $48.15 \%, \mathrm{G} / \mathrm{G}: 14.44 \%, p=0.006$ ) (Table 2 ). In contrast, $A P O A 1$ rs670 was not associated with tumor occurrence side, type, size, and stage or a family history (Table 2). The odds of being lymph node-positive in APOA1 rs670 G/A carrying patients $(\mathrm{OR}=3.32,95 \% \mathrm{CI}=1.77-6.20, p<$ 0.001 ) and $A P O A 1$ rs670 A/A carriers (OR=2.58, 95\% $\mathrm{CI}=1.05-6.32, p=0.039)$ was higher than $\mathrm{G} / \mathrm{G}$ carriers (Table 3). On the other hand, APOA1 rs670 A/A carriers have significantly higher odds in having ER/PR doublenegative tumors than their $\mathrm{G} / \mathrm{G}$ counterparts $(\mathrm{OR}=4.85$, 95\% CI=1.83-12.83, $p=0.001$ ) (Table 3).

\section{APOA1 rs670 A allele carriage was associated with poor post-surgery outcomes}

Higher proportions of APOA1 rs670 G/A and A/A carrying breast cancer patients developed recurrence or death than G/G carriers (Table 4). The higher incidences of recurrence in APOA1 rs670 G/A and A/A carriers translate higher risks to 2.07 -fold ( $95 \% \mathrm{CI}=1.03-4.14, p=0.041)$ and 3.44-fold ( $95 \% \mathrm{CI}=1.33-8.86, p=0.011)$ risk as compared with G/G carriers, respectively (Table 5 ). However, the increase in risk of recurrence in APOA1 rs670 G/A carriers may be confounded by their increased odds in worse baseline phenotype. After adjustment for lymph node status, the statistical significance remained in A/A carriers $(\mathrm{OR}=2.83,95 \% \mathrm{CI}, 1.05-7.61, p=0.040)$, while that in G/A carriers were diminished $(p=0.238)$ (Table 5). The risk of death was significantly increased in APOA1 rs670 A/A carriers $(\mathrm{OR}=6.03,95 \% \mathrm{CI}=2.08-17.51, p<0.001)$ and this persisted after adjustments for baseline confounders of lymph node $(p=0.007)$ and ER/PR $(p=0.010)$ status, while no significant risk was observed in G/A carriers with or without adjustments (Table 5). The mean years in recurrence-free survival and overall survival of $A P O A 1$ rs670 A/A carriers were significantly shorter than that of G/A and G/G counterparts (all $p<0.001$ ) (Table 4). Despite 
Table 2 APOA1 rs670 A allele carriage was associated with lymph node and hormone receptor status at baseline

\begin{tabular}{|c|c|c|c|c|}
\hline & $\mathrm{G} / \mathrm{G}(\mathrm{n}=90)$ & $G / A(n=106)$ & $A / A(n=27)$ & $P$ value $^{a}$ \\
\hline Age & $46.61 \pm 10.14(29.25-74.55)$ & $49.98 \pm 10.24(29.87-74.57)$ & $47.83 \pm 9.81(29.25-67.91)$ & 0.067 \\
\hline $\mathrm{BMI}^{\mathrm{b}}$ & $23.47 \pm 4.14(15.06-34.48)$ & $23.03 \pm 4.16(2.25-38.05)$ & $23.77 \pm 3.83(18.18-32.05)$ & 0.654 \\
\hline \multicolumn{5}{|l|}{ Tumor type } \\
\hline Infiltrating ductal carcinoma & $73(81.11 \%)$ & 97 (91.51\%) & $23(85.19 \%)$ & \multirow{4}{*}{0.332} \\
\hline Ductal carcinoma in situ & $10(11.11 \%)$ & $5(4.72 \%)$ & $2(7.41 \%)$ & \\
\hline Others & $5(5.56 \%)$ & $4(3.77 \%)$ & $2(7.41 \%)$ & \\
\hline Unknown & $2(2.22 \%)$ & $0(0.00 \%)$ & $0(0.00 \%)$ & \\
\hline \multicolumn{5}{|l|}{ Side } \\
\hline Right & $35(38.89 \%)$ & $52(49.06 \%)$ & $13(48.15 \%)$ & \\
\hline Left & $51(56.67 \%)$ & $50(47.17 \%)$ & $13(48.15 \%)$ & 0.704 \\
\hline Bilateral & $4(4.44 \%)$ & $4(3.77 \%)$ & $1(3.70 \%)$ & \\
\hline \multicolumn{5}{|l|}{ Tumor size } \\
\hline$<5 \mathrm{~cm}$ & $64(71.11 \%)$ & $76(71.70 \%)$ & 19 (70.37\%) & \\
\hline$\geq 5 \mathrm{~cm}$ & $6(6.67 \%)$ & $16(15.09 \%)$ & $4(14.81 \%)$ & 0.227 \\
\hline Unknown & $20(22.22 \%)$ & $14(13.21 \%)$ & $4(14.81 \%)$ & \\
\hline \multicolumn{5}{|l|}{ Lymph node involvement } \\
\hline Positive & $22(24.44 \%)$ & $55(51.89 \%)$ & $13(48.15 \%)$ & \\
\hline Negative & $61(67.78 \%)$ & $46(43.40 \%)$ & $14(51.85 \%)$ & 0.002 \\
\hline Unknown & 7 (7.78\%) & $5(4.72 \%)$ & $0(0.00 \%)$ & \\
\hline \multicolumn{5}{|l|}{ TMN staging } \\
\hline Stages $0-2$ & $63(70.00 \%)$ & $71(66.98 \%)$ & $20(74.07 \%)$ & \\
\hline Stages $>2$ & $7(7.78 \%)$ & 19 (17.92\%) & $4(14.81 \%)$ & 0.194 \\
\hline Unknown & $20(22.22 \%)$ & $16(15.09 \%)$ & $3(11.11 \%)$ & \\
\hline \multicolumn{5}{|l|}{ ER/PR status } \\
\hline Single or double positive & $63(70.00 \%)$ & $68(64.15 \%))$ & $13(48.15 \%)$ & \\
\hline Negative & $13(14.44 \%)$ & $26(24.53 \%)$ & $13(48.15 \%)$ & 0.006 \\
\hline Unknown & $14(15.56 \%)$ & $12(11.32 \%)$ & 1 (3.70\%) & \\
\hline \multicolumn{5}{|l|}{ Family history } \\
\hline Positive & $4(4.44 \%)$ & $3(2.83 \%)$ & $(0.00 \%)$ & \\
\hline Negative & $81(90.00 \%)$ & 97 (91.51\%) & $25(92.59 \%)$ & 0.823 \\
\hline Unknown & $5(5.56 \%)$ & $6(5.66 \%)$ & $2(7.41 \%)$ & \\
\hline
\end{tabular}

NOTE: Data shown represent number of patients (\% of $\mathrm{n}$ ) or mean \pm s.d.. Bold type indicates $p<0.050$.

a, results of Chi-squared analysis or ANOVA test, depending on the type of parameter analyzed.

, results from 185 patients.

Abbreviations: $E R$ estrogen receptor, $P R$ progesterone receptor.

the significance in increased recurrence risk, the $A P O A 1$ rs670 G/A breast cancer carriers had mean survival years comparable to their $\mathrm{G} / \mathrm{G}$ counterparts.

\section{APOA1 rs670 A/A carriers had the worst outcome in lymph node-negative patients}

To compare APOA1 rs670 A/A carriage with the other known risk factors in post-surgery prognosis, we performed Cox regression analysis including ER/PR status, lymph node involvement, age, BMI and post-surgery adjuvant in adjusted models. Of specific importance, lymph node-positivity, ER/PR-negativity, combined therapy, and APOA1 rs670 A/A carriage showed increased recurrence and mortality risk in unadjusted models (Table 6). The increased risk of recurrence and mortality observed in APOA1 rs670 A/A carriers remained significant after adjustments for lymph node involvement, ER/PR status, age, BMI, or post-surgery adjuvant (Additional file 4). When testing the prognostic effects of lymph node, ER/PR, postsurgery adjuvant and $A P O A 1$ rs670 A/A carrying status in an adjusted model, the significance of lymph node and APOA1 rs670 A/A was stronger than of ER/PR and postsurgery adjuvant (Table 6). Lymph node positivity showed 
Table 3 APOA1 rs670 A allele carrying patients had increased odds of lymph node involvement and hormone receptor expression negativity at baseline

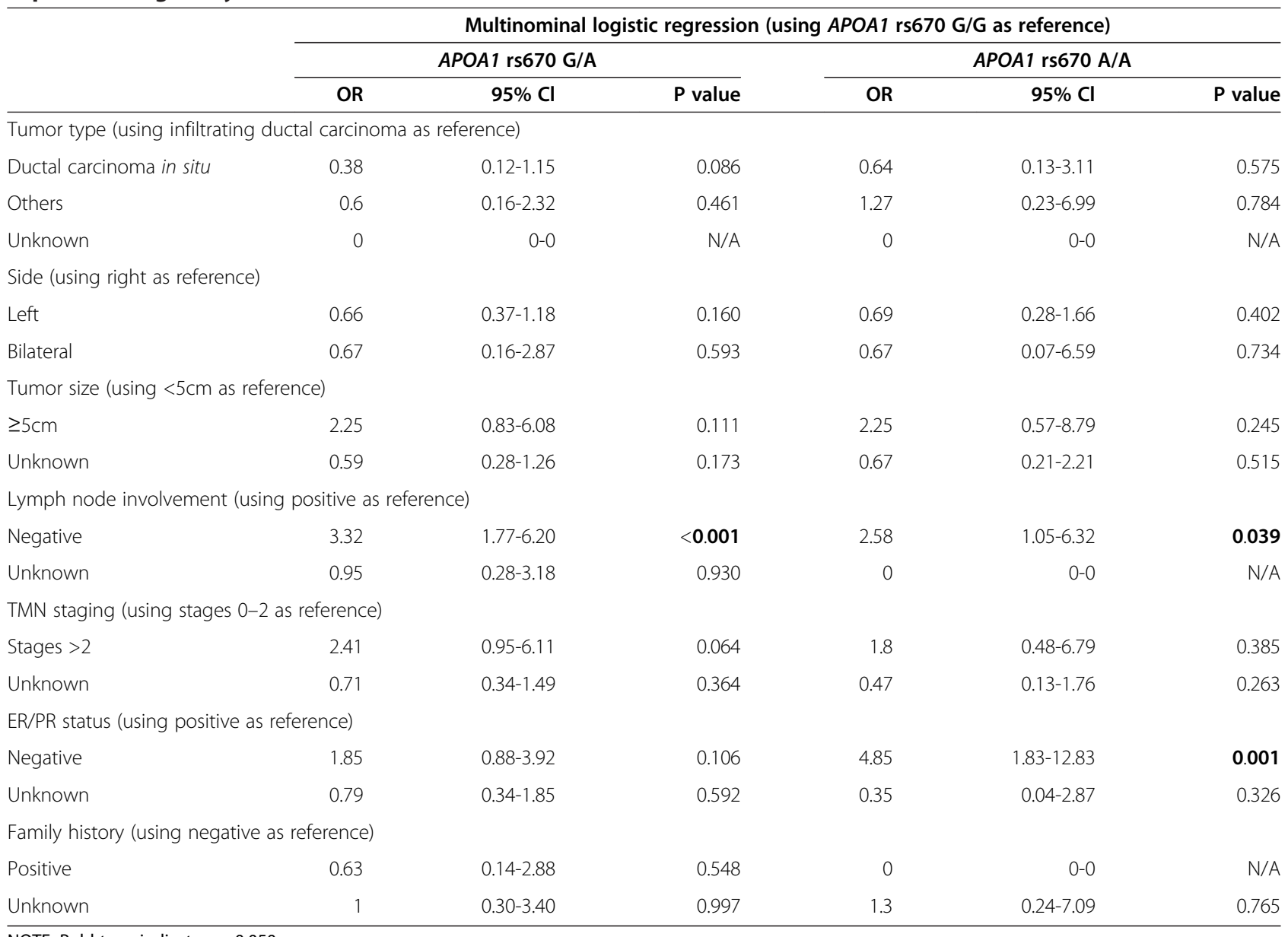

NOTE: Bold type indicates $p<0.050$.

Abbreviations: $C l$ confidence interval, $E R$ estrogen receptor, $P R$ progesterone receptor, $O R$ odds ratio.

increased risks of recurrence $(\mathrm{HR}=2.62,95 \% \mathrm{CI}=1.43-4.79$, $p=0.002)$ and mortality $(\mathrm{HR}=5.35,95 \% \mathrm{CI}=2.25-12.68$, $p<0.001$ ), while APOA1 rs670 A/A carriage in recurrence $(\mathrm{HR}=3.12,95 \% \mathrm{CI}=1.29-7.56, p=0.012)$ and mortality $(\mathrm{HR}=4.36,95 \% \mathrm{CI}=1.52-12.47, p=0.006)$. In contrast, $\mathrm{ER} /$ PR status was associated with mortality but no longer with recurrence in the adjusted model, while post-surgery adjuvant shows a reversed pattern (Table 6).
Therefore, to specifically rule out confounding from baseline lymph node involvement in predicting prognosis, we tested the prognostic effect of APOA1 rs670 A/A carriage in baseline lymph node status-stratified patient groups. The prognostic effect of APOA1 rs670 A/A carriage was only observed in the lymph node-negative patients $(\mathrm{n}=121$, Table 7 and Figure 1$)$ with higher hazard risks than non-stratified group $(n=223$, Table 4 and

Table 4 APOA1 rs670 A allele carriage was associated with poor post-surgery outcomes

\begin{tabular}{|c|c|c|c|c|}
\hline & $\mathrm{G} / \mathrm{G}(\mathrm{n}=90)$ & $G / A(n=106)$ & $A / A(n=27)$ & $P$ value $^{\mathrm{a}}$ \\
\hline Recurrence & $15(16.67 \%)$ & $30(28.30 \%)$ & $11(40.74 \%)$ & 0.021 \\
\hline Mortality & $8(8.89 \%)$ & $15(14.15 \%)$ & $10(37.04 \%)$ & 0.002 \\
\hline \multicolumn{5}{|c|}{ Mean survival years } \\
\hline Recurrence-free & $9.63 \pm 4.97(0.16-21.38)$ & $10.03 \pm 5.45(0.04-24.52)$ & $5.36 \pm 4.11(0.18-17.21)$ & $<0.001$ \\
\hline Overall & $10.30 \pm 4.67(0.16-21.38)$ & $10.98 \pm 5.32(0.14-24.52)$ & $6.33 \pm 4.00(0.55-17.21)$ & $<0.001$ \\
\hline
\end{tabular}

NOTE: Data shown represent number of patients (\% of $\mathrm{n}$ ) or mean \pm s.d.. Bold type indicates $p<0.050$.

${ }^{a}$, results of Chi-squared analysis or ANOVA test, depending on the type of parameter analyzed.

Abbreviations: $E R$ estrogen receptor, $P R$ progesterone receptor. 
Table 5 APOA1 rs670 A allele carrying patients had increased odds of post-surgery disease progression

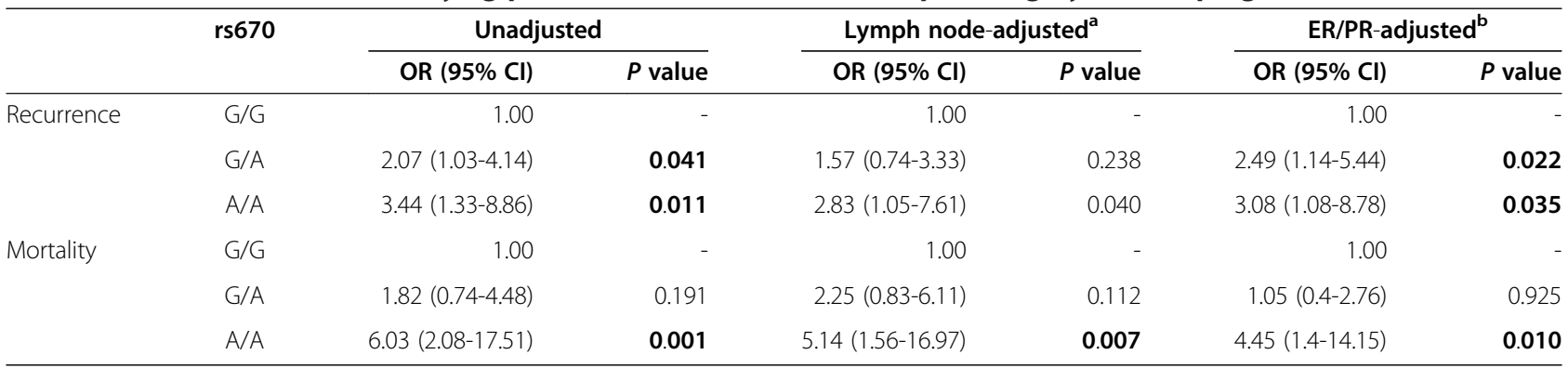

NOTE: Data shown represent number of patients (\% of $n$ ) or mean \pm s.d.. Bold type indicates $p<0.050$.

, adjusted for lymph node status, $\mathrm{n}=211$.

, adjusted for ER/PR status, $\mathrm{n}=196$.

Abbreviations: $C l$ confidence interval, $E R$ estrogen receptor, $P R$ progesterone receptor, $O R$ odds ratio.

Additional file 5 upper panels). The prognostic effect of APOA1 rs670 A/A carriage did not reach statistical significance in node-positive patients $(\mathrm{n}=90$, Table 7 and Additional file 5 lower panels). The risk of recurrence associated with $A P O A 1$ rs670 A/A carriage was higher as compared with $\mathrm{G} / \mathrm{G}(\mathrm{HR}=4.98,95 \% \mathrm{CI}=1.40-17.70$, $p=0.013)$ in lymph node-negative breast cancer patients. The risk of mortality in lymph node-negative $A P O A 1$ rs670 A/A carrying breast cancer patients was also higher than $A P O A 1 \mathrm{rs} 670 \mathrm{G} / \mathrm{G}$ counterparts (HR=9.87, 95\% $\mathrm{CI}=1.60-60.81, p=0.014)$. The contributions from ER/PR status, age, BMI, or post-surgery adjuvant in predicting outcome remained insignificant in lymph node-status stratified groups (Table 7).

\section{Discussion}

Dyslipidemia is a known complication of TAM-treatment in breast cancer patients, and increases risk of coronaryartery diseases in patients [33]. Of equal importance, dyslipidemia has been observed in breast cancer patients prior to treatment, and this correlation is supported by epidemiological studies [8-11]. Concomitantly, usage of lipid-lowering drugs such as statin and niacin has been shown to associate with decreased breast cancer recurrence

Table 6 APOA1 rs670 A/A carriage predicts poor post-surgery survival in breast cancer after adjustments for lymph node and ER/PR status

\begin{tabular}{|c|c|c|c|c|}
\hline & \multicolumn{2}{|c|}{ Unadjusted } & \multicolumn{2}{|c|}{ Adjusted $(n=186)$} \\
\hline & HR $(95 \% \mathrm{Cl})$ & $P$ value & HR $(95 \% \mathrm{Cl})$ & $P$ value \\
\hline \multicolumn{5}{|l|}{ Recurrence } \\
\hline Lymph node positivity ${ }^{a}$ & $2.86(1.65-4.96)$ & $<0.001$ & $2.20(1.18-4.10)$ & 0.013 \\
\hline ER/PR negativity ${ }^{b}$ & $1.90(1.08-3.37)$ & 0.027 & $1.53(0.85-2.77)$ & 0.156 \\
\hline Age & $1.01(0.98-1.04)$ & 0.479 & - & - \\
\hline $\mathrm{BMI}^{\mathrm{C}}$ & $1.00(0.93-1.08)$ & 1.000 & - & - \\
\hline Combined therapy $^{d}$ & $2.64(1.46-4.78)$ & 0.001 & $2.03(1.01-4.08)$ & 0.046 \\
\hline APOA1 rs670 A/A $\mathrm{A}^{\mathrm{e}}$ & $4.02(2.22-9.96)$ & 0.001 & $3.02(1.25-7.29)$ & 0.014 \\
\hline \multicolumn{5}{|l|}{ Mortality } \\
\hline Lymph node positivity ${ }^{\mathrm{a}}$ & $6.01(2.62-13.80)$ & $<0.001$ & $2.09(1.01-4.29)$ & 0.046 \\
\hline ER/PR negativity ${ }^{b}$ & $2.30(1.13-4.70)$ & 0.022 & $4.54(1.88-10.94)$ & 0.001 \\
\hline Age & $1.03(0.99-1.06)$ & 0.127 & - & - \\
\hline $\mathrm{BMI}^{\mathrm{C}}$ & $1.00(0.91-1.10)$ & 0.942 & - & - \\
\hline Combined therapy ${ }^{d}$ & $3.45(1.50-7.98)$ & 0.004 & $2.11(0.84-5.31)$ & 0.114 \\
\hline APOA1 rs670 A/A $\mathrm{A}^{\mathrm{e}}$ & $6.30(2.47-16.08)$ & $<0.001$ & $4.47(1.56-12.79)$ & 0.005 \\
\hline
\end{tabular}

NOTE: $P$ values were results of Cox proportional hazard regression analysis. Bold type indicates $p<0.050$.

a, compared with negative lymph node involvement, $\mathrm{n}=211$.

b' compared with ER/PR positive, $\mathrm{n}=196$.

c, $\mathrm{n}=185$.

d, compared with TAM only.

e, compared with APOA $1 \mathrm{rs} 670 \mathrm{G} / \mathrm{G}$.

Abbreviations: $B M I$ Body mass index, $E R$ estrogen receptor, $H R$ Hazard ratio, $P R$ progesterone receptor. 
Table 7 APOA1 rs670 A/A carriers has the worst post-surgery outcomes in lymph node-negative patients

\begin{tabular}{|c|c|c|c|c|c|c|}
\hline & \multicolumn{6}{|c|}{ Cox proportional hazard regression } \\
\hline & \multicolumn{3}{|c|}{ Lymph node negative $(n=121)$} & \multicolumn{3}{|c|}{ Lymph node positive $(n=90)$} \\
\hline & HR & $95 \% \mathrm{Cl}$ & $P$ value & HR & $95 \% \mathrm{Cl}$ & $P$ value \\
\hline \multicolumn{7}{|l|}{ Recurrence } \\
\hline ER/PR negativity ${ }^{a}$ & 2.06 & $0.80-5.31$ & 0.136 & 0.62 & $0.30-0.62$ & 0.202 \\
\hline Age & 1.01 & $0.96-1.05$ & 0.788 & 1.01 & $0.98-1.01$ & 0.582 \\
\hline BMI & 0.95 & $0.84-1.08$ & 0.450 & 1.02 & $0.92-1.02$ & 0.703 \\
\hline Combined therapy ${ }^{b}$ & 2.02 & 0.83-4.93 & 0.120 & 2.12 & $0.85-5.29$ & 0.107 \\
\hline APOA1 rs670 A/A $\mathrm{A}^{\mathrm{C}}$ & 4.98 & $1.40-17.70$ & 0.013 & 2.46 & $0.86-2.46$ & 0.093 \\
\hline \multicolumn{7}{|l|}{ Mortality } \\
\hline ER/PR negativity ${ }^{a}$ & 0.24 & $0.05-1.08$ & 0.064 & 0.52 & $0.22-1.18$ & 0.118 \\
\hline Age & 1.05 & $0.98-1.13$ & 0.196 & 1.02 & $0.99-1.06$ & 0.262 \\
\hline BMl & 0.89 & $0.71-1.11$ & 0.296 & 1.03 & $0.91-1.16$ & 0.648 \\
\hline Combined therapy ${ }^{b}$ & 1.83 & $0.41-8.18$ & 0.430 & 2.65 & $0.89-7.88$ & 0.081 \\
\hline APOA1 rs670 A/A $\mathrm{A}^{\mathrm{C}}$ & 9.87 & $1.60-60.81$ & 0.014 & 2.86 & $0.96-8.55$ & 0.060 \\
\hline
\end{tabular}

and risk [15-17]. SNPs on the $A P O A 1 / C 3 / A 5$ gene cluster, which is involved in lipid metabolism, are highly associated with dyslipidemia, metabolic syndrome, insulin resistance and responsiveness to relative treatments [19]. However, the contribution of SNPs on the $A P O A 1 / C 3 / A 5$ gene cluster to breast cancer is yet to be defined. In this study, we tested the effects of dyslipidemia-associated SNPs on the APOA1/C3/A5 gene cluster: APOA1 rs670, APOC3 rs2854116, APOC3 rs2854117, APOA5 rs662799 and APOA5 rs2075291 on breast cancer progression in a Taiwanese patient group with mainly operable early-stage tumors, and a 10-year follow-up interval. We showed that only APOA 1 rs670 out of the 5 tested SNPs was correlated to breast cancer, lymph node-positivity, ER/PR doublenegativity at baseline. Furthermore, carriers of both minor alleles on APOA1 rs670 had shortest survival time and highest risk in disease progression independent of baseline characteristics. Moreover, the prognostic value of $A P O A 1$ rs670 A/A carriage in the worse post-surgery outcomes was most evident in lymph node-negative patients.

The rs670 SNP contains a G-to-A substitution at 75bp upstream of $A P O A 1$ transcriptional start site, and is within a MSPI restriction enzyme recognition [19]. A cross-sectional study focusing on the association of APOA1 SNPs with breast cancer and patient phenotype reported that the $-75 \mathrm{G} / \mathrm{A}$ polymorphism correlated with breast cancer risk at baseline [34]. However, the slight discrepancy in associations of -75G/A polymorphism with ER status observed by us and Hamrita et al. is likely due to ethnic (Taiwanese vs. Tunisian) and age differences $(62.7 \%$ vs. $38.9 \%<50$ years $)$ [34]. Nevertheless, in our longitudinal study we found that $A P O A 1$ rs670 predicted worse outcome after adjustment for ER/PR status, indicating alternative contributions from APOA1 rs670 in cancer progression. ApoA1 is the structural protein of HDL, and interacts with lecithin cholesterol acyl transferase, which controls the limiting steps in HDL maturation and therefore reverse cholesterol transport [19]. The minor allele of APOA1 rs670 has been correlated to altered HDL-C, diabetes and coronaryartery disease: lower levels of HDL-C was observed in Northern Indians [20], and correlated with severe forms of cardiovascular diseases in Northern Indians, Caucasians in Spain and Australia [20,24,25]. Moreover, APOA1 rs670 A/A Spanish carriers had a higher risk of diabetes than their non-A/A counterparts, though their HDL-C did not differ significantly [21,22]. As plasma apoA1 level is regulated by estrogen and thus TAM treatments, the timing of plasma apoA1 measurement in breast cancer patients is critical. However, we did not have access to TAM treatment-naïve plasma of the patients recruited in this study. Nevertheless, the disease phenotype associated $A P O A 1$ rs670 remains significant without HDL-C differences, as has been reported in a number of studies on metabolic/cardiovascular diseases $[21,24,25]$.

The significance of plasma HDL-C levels in breast cancer has long been investigated and debated [35]. The current held view is that higher HDL-C levels, which are preventive for cardiovascular events, are also protective for breast cancer. The majority of reports supporting this view were of case-control design, and found lower 


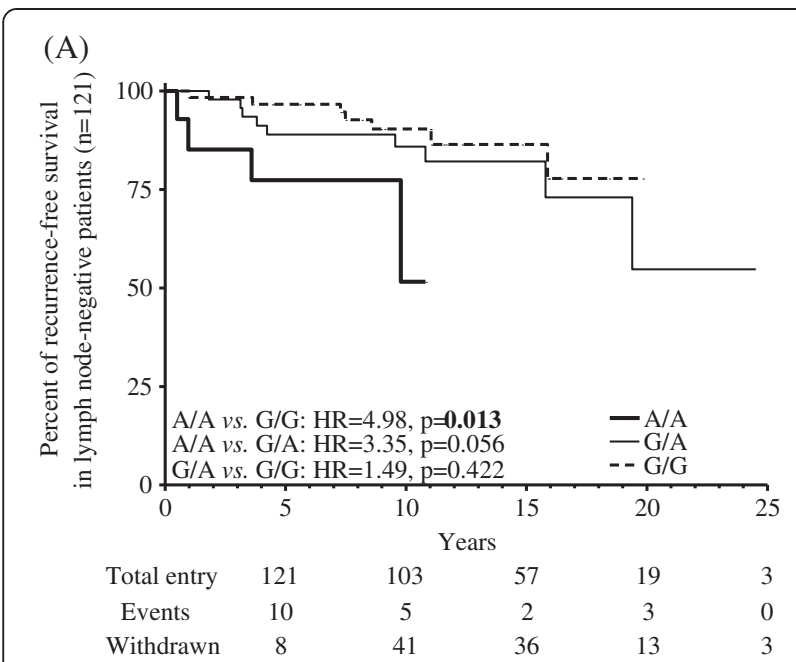

(B)

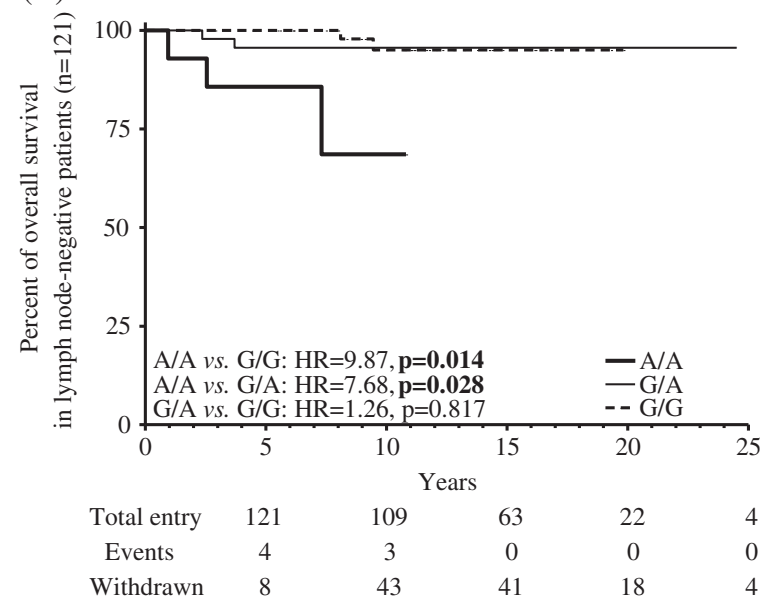

Figure 1 Kaplan-Meier plots of breast cancer patient post-surgery survival stratified by $A P O A 1$ rs 670 genotype. The recurrence-free $(\mathbf{A})$ and overall disease-specific survival (B) of APOA1 A/A (bold line), G/A (thin line) and G/G (broken line) carrying lymph-node negative breast cancer patients were compared. The life tables are shown below the Kaplan-Meier survival plots. Bold type indicates $p<0.050$.

total or lipid-rich HDL, or higher total cholesterol/HDL$\mathrm{C}$ ratio in breast cancer patients as compared with noncancerous or other cancer controls in various ethnic groups [8,10,11]. A 17.2-year prospective cohort study carried out in Norway found that naive females with the lowest quartile of HDL-C had highest risk of later developing breast cancer [14]. Moreover, the same Norwegian group found the breast cancer patients with highest total cholesterol or lowest plasma HDL-C levels had highest risk in mortality [13]. The female breast is an active site of both lipid uptake and lipid secretion during periods of milk secretion, thus it is readily acceptable that apoA1 is present in human milk [36]. Supportively, apoA1 was also identified in the breast cancer tissues and ex vivo cultured medium. ApoA1 was found in breast tumors, and its amount was correlated positively with chemotherapy resistance in malignant tumors [37]. In contrast, breast tumors later found responsive to chemotherapy secreted higher amount of apoA1 than tumors nonresponsive to chemotherapy during short term ex vivo culture, while non-cancerous tissues secreted highest amount of apoA1 [38]. Furthermore, a successful reduction of breast cancer growth in mice by vitamin D treatment was accompanied by decrease of apoA1 production in tumor tissues [39]. It is at this stage unknown how much apoA1 in circulation is contributed by the mammary tissue, but the retention of apoA1 in breast cancer tissues is in accordance with the observation of lower plasma HDL/apoA1 in breast cancer patients. Though the association of plasma lipid profiles and breast cancer incidence or progression is strongly and continuously suggested, the molecular mechanisms of HDL or apoA1 in promoting breast cancer risk are still unclear.

Plasma apoA1/lipid-poor HDL bind to ATP-binding cassette $(\mathrm{ABC})$ lipid transporters during maturation, and the intracellular cholesterol is exported to load onto HDL, facilitating reverse cholesterol transport [40]. Several members of the $A B C$ family were found in mammary tissues, but only those associated with drug resistance were increased in breast cancers as compared with normal tissues [41]. In contrast, $\mathrm{ABC}$ transporters involved in lipid transport, including $A B C-A 1$ and $A B C$ $\mathrm{A} 3$, were decreased in breast cancer tissues as compared with healthy mammary glands [42]. In vitro studies found that over-expression of ABC-G1 in breast cancer cell line induces cellular cholesterol efflux and apoptosis [43]. Moreover, ABCA- or ABCG-HDL binding concomitantly induces intracellular signaling and suppresses inflammatory reactions, which is known to be a key factor in tumorigenesis. Binding of ABCA or ABCG by apoA1 induces the recruitment of phosphrylated STAT3 and JAK2, and thus suppresses the inflammatory pathways including TL4-signalling [40]. Furthermore, ABCA induces Cdc42 and PAK-1 signaling, which in turn are regulative for breast cancer ER/PR expression in vitro and in vivo $[44,45]$. Therefore, the altered HDL/apoA1 levels observed in APOA1 rs670 A carriers may participate in the tumorigenesis, survival, ER/PR status of breast cancer cells. In a previous published study, we found that genetic and intra-tumoral ER polymorphisms were correlated with breast cancer in Taiwanese females [30,31]. It would be of interest how $A P O A 1$ rs670 and HDL-C interact with ER polymorphisms both at a systemic and local (mammary tissue) level in these patients.

Clinically, our finding of worst outcome in APOA1 rs670 A/A breast cancer patients as compared to their non-A/A counterparts provides pivotal implications in personalized treatment regimen and therapeutic strategies. The median 
recurrence-free and overall survival years of $A P O A 1 \mathrm{rs} 670$ A/A carrying breast cancer patients were both longer than the standard chemotherapy duration ( 1 year) and the advised 5-year limit for continuous TAM usage. As the patients recruited in this study were non-obese and of early tumor-staging, the administration of adjuvant therapy may be less intensive. Furthermore, the prognostic value of APOA1 rs670 was even more evident in the lymph nodenegative patients, who were given less adjuvant therapies under the current assessment guidelines. Thus APOA1 rs670 A/A carriers may benefit from advanced therapies. Recent studies demonstrated that administration of apoA1 mimetic peptides reduced the development of cancer in murine models [46,47]. Furthermore, a large cohort study on the effect of post-diagnosis statin use in breast cancer females reported a lowered risk in cancer recurrence [48]. These reports suggest that raising HDL-C or apoA1 may be preventive or therapeutic for breast cancers, especially in the $A P O A 1 \mathrm{rs} 670 \mathrm{~A} / \mathrm{A}$ carrying patients. On the other hand, due to the low plasma HDL-C and tumor ER/PR negativity in the APOA1 rs670 A/A carriers, the usage of TAM in these breast cancer patients may be controversial and require further evaluation.

\section{Conclusions}

In conclusion, we demonstrated the correlation of dyslipidemia-associated APOA1 rs670 minor allele with unfavorable baseline characteristics in Taiwanese breast cancer patients, and the 10-year follow-up revealed patients carrying both minor alleles had worst survival in lymph node-negative patients. Though TAM treatment naïve plasma HDL-C levels of the recruited patients were unavailable, our data was consistent after appropriate adjustments for possible confounding factors. Our study provides novel clinical directions for both personalized treatment regimen and therapeutic strategies.

\section{Additional files}

Additional file 1: The distributions of tested SNPs on APOA1/C3/A5 gene cluster in breast cancer patients and healthy controls.

Additional file 2: The linkage disequilibrium pattern of tested APOA1/C3/A5 SNP gene cluster in the study group. The SNP linkage pattern in healthy controls (A) and breast cancer patients (B) are shown. The upper panels show the relative chromosomal localization of the five SNPs, and the lower panels the test results of linkage disequilibrium by Haploview. The colour scale shown in the lower panels demonstrates high linkage disequilibrium (red) to minimal linkage disequilibrium (white). The numbers in the lower panels represent the pair-wise $\mathrm{D}^{\prime}$ values which are shown in two digits after the decimal point. Linkage tests with $L O D$ values $\geq 2$ and $D^{\prime}$ values $>0.80$ were considered as presence of significant linkage. $D^{\prime}$ values of 1.00 are not shown.

Additional file 3: The allele frequencies of tested APOA1/C3/A5 SNPs in breast cancer patients and healthy controls.

Additional file 4: The comparison of APOA1 rs670 A/A carriage with other risk factors in predicting disease progression.
Additional file 5: The Kaplan-Meier survival plots of APOA1 rs670 genotype carrying breast cancer patients. The recurrence-free ( $\mathbf{A}$ and C) and overall disease-specific survival (B and $\mathbf{D}$ ) of APOA1 A/A (bold line), $G / A$ (thin line) and $G / G$ (broke line) carrying breast cancer patients were compared. Data from all recruited patients $(n=223)$ are shown in $A$ \& $B$, and lymph-node positive patients $(n=90)$ in $\mathbf{C} \& \mathbf{D}$. The life tables are shown below the Kaplan-Meier plots.

\section{Abbreviations}

HDL-C: High density lipoprotein-cholesterol; Apo: Apolipoproteins; SNP: Single-nucleotide polymorphisms; BMI: Body mass index; ER: Estrogen receptor; PR: Progesteron receptor; TAM: Tamoxifen.

\section{Competing interests}

The authors declare that they have no competing interests.

\section{Author contributions}

$\mathrm{MCH}, \mathrm{WCH}$, and $\mathrm{KCY}$ conceived and designed the study. MCH and KCY developed the methodology, performed laboratory experiments, and supervised the study. MCH, WCH, and KTL acquired and prepared clinical data. MCH, WCH, KTL, CHW, HYS, ILL and KCY analyzed and interpreted the data. $M C H, W C H, K T L, C H W, H Y S$, ILL and KCY wrote, reviewed and edited the manuscript. All authors read and approved the final manuscript.

\section{Acknowledgments}

We would like to thank Miss Ting-Yu Hou and Miss I-Chun Shih for technical support and assistance. This study is supported by the National Science Council, Taiwan (grant no. NSC 100-2321-B-006-015-MY3, and NSC 100-2320 -B-006-007-MY3). This article is prepared in memory of the late Professor Ping-Wen Lin (1951-2012).

\section{Author details}

${ }^{1}$ Research Center for Medical Laboratory Biotechnology, College of Medicine, National Cheng Kung University, Tainan, Taiwan. ${ }^{2}$ Department of Medical Laboratory Science and Biotechnology, College of Medicine, National Cheng Kung University, Tainan, Taiwan. ${ }^{3}$ Department of Surgery, College of Medicine, National Cheng Kung University, Tainan, Taiwan. ${ }^{4}$ Department of Surgery, Yang Ming Hospital, Chiayi, Taiwan. ${ }^{5}$ Department of Family Medicine, College of Medicine, National Cheng Kung University, Tainan, Taiwan. ${ }^{6}$ Department of Medical Laboratory Science and Biotechnology, College of Health Sciences, Kaohsiung Medical University, Kaohsiung, Taiwan. ${ }^{7}$ Center of Infectious Disease and Signaling Research, College of Medicine, National Cheng Kung University, Tainan, Taiwan.

Received: 22 March 2013 Accepted: 30 June 2013

Published: 5 July 2013

\section{References}

1. Hutchinson L: Breast cancer: challenges, controversies, breakthroughs. Nat Rev Clin Oncol 2010, 7(12):669-670.

2. Oakman C, Santarpia L, Di Leo A: Breast cancer assessment tools and optimizing adjuvant therapy. Nat Rev Clin Oncol 2010, 7(12):725-732.

3. Cheng SH, Tsou MH, Liu MC, Jian JJ, Cheng JC, Leu SY, Hsieh CY, Huang AT: Unique features of breast cancer in Taiwan. Breast Cancer Res Treat 2000, 63(3):213-223.

4. Huang $C S$, Lin $C H$, Lu YS, Shen CY: Unique features of breast cancer in Asian women-breast cancer in Taiwan as an example. I Steroid Biochem Mol Biol 2010, 118(4-5):300-303.

5. Champ CE, Volek JS, Siglin J, Jin L, Simone NL: Weight gain, metabolic syndrome, and breast cancer recurrence: are dietary recommendations supported by the data? Int J Breast Cancer 2012, 2012:506868.

6. La Vecchia C, Giordano SH, Hortobagyi GN, Chabner B: Overweight, obesity, diabetes, and risk of breast cancer: interlocking pieces of the puzzle. Oncologist 2011, 16(6):726-729.

7. Eckel RH, Alberti KG, Grundy SM, Zimmet PZ: The metabolic syndrome. Lancet 2010, 375(9710):181-183.

8. Chang SJ, Hou MF, Tsai SM, Wu SH, Hou LA, Ma H, Shann TY, Tsai LY: The association between lipid profiles and breast cancer among Taiwanese women. Clin Chem Lab Med 2007, 45(9):1219-1223. 
9. Goodwin PJ, Boyd NF, Hanna W, Hartwick W, Murray D, Qizilbash A, Redwood S, Hood N, DelGiudice ME, Sidlofsky S, et al: Elevated levels of plasma triglycerides are associated with histologically defined premenopausal breast cancer risk. Nutr Cancer 1997, 27(3):284-292.

10. Kim Y, Park SK, Han W, Kim DH, Hong YC, Ha EH, Ahn SH, Noh DY, Kang D, Yoo KY: Serum high-density lipoprotein cholesterol and breast cancer risk by menopausal status, body mass index, and hormonal receptor in Korea. Cancer Epidemiol Biomarkers Prev 2009, 18(2):508-515.

11. Michalaki V, Koutroulis G, Syrigos K, Piperi C, Kalofoutis A: Evaluation of serum lipids and high-density lipoprotein subfractions (HDL2, HDL3) in postmenopausal patients with breast cancer. Mol Cell Biochem 2005, 268(1-2):19-24.

12. Bahl M, Ennis M, Tannock IF, Hux JE, Pritchard KI, Koo J, Goodwin PJ: Serum lipids and outcome of early-stage breast cancer: results of a prospective cohort study. Breast Cancer Res Treat 2005, 94(2):135-144.

13. Emaus A, Veierod MB, Tretli S, Finstad SE, Selmer R, Furberg AS, Bernstein L, Schlichting E, Thune I: Metabolic profile, physical activity, and mortality in breast cancer patients. Breast Cancer Res Treat 2010, 121(3):651-660.

14. Furberg AS, Veierod MB, Wilsgaard T, Bernstein L, Thune I: Serum highdensity lipoprotein cholesterol, metabolic profile, and breast cancer risk. J Natl Cancer Inst 2004, 96(15):1152-1160.

15. Shrubsole MJ, Shu XO, Li HL, Cai H, Yang G, Gao YT, Gao J, Zheng W: Dietary $B$ vitamin and methionine intakes and breast cancer risk among Chinese women. Am J Epidemiol 2011, 173(10):1171-1182.

16. Fagherazzi G, Fabre A, Boutron-Ruault MC, Clavel-Chapelon F: Serum cholesterol level, use of a cholesterol-lowering drug, and breast cancer: results from the prospective E3N cohort. Eur J Cancer Prev 2010, 19(2):120-125.

17. Ahern TP, Pedersen L, Tarp M, Cronin-Fenton DP, Garne JP, Silliman RA, Sorensen HT, Lash TL: Statin prescriptions and breast cancer recurrence risk: a Danish nationwide prospective cohort study. J Natl Cancer Inst 2011, 103(19):1461-1468

18. Alikhani N, Ferguson RD, Novosyadlyy R, Gallagher EJ, Scheinman EJ, Yakar S, Leroith D: Mammary tumor growth and pulmonary metastasis are enhanced in a hyperlipidemic mouse model. Oncogene 2013, 32:961-967.

19. Lai CQ, Parnell LD, Ordovas JM: The APOA1/C3/A4/A5 gene cluster, lipid metabolism and cardiovascular disease risk. Curr Opin Lipidol 2005 16(2):153-166

20. Chhabra S, Narang R, Lakshmy R, Das N: APOA1-75 G to A substitution associated with severe forms of CAD, lower levels of HDL and apoA-I among northern Indians. Dis Markers 2005, 21(4):169-174.

21. Ma YQ, Thomas GN, Ng MC, Critchley JA, Cockram CS, Chan JC, Tomlinson $\mathrm{B}$ : Association of two apolipoprotein A-I gene Mspl polymorphisms with high density lipoprotein (HDL)-cholesterol levels and indices of obesity in selected healthy Chinese subjects and in patients with early-onset type 2 diabetes. Clin Endocrinol (Oxf) 2003, 59(4):442-449.

22. Morcillo S, Cardona F, Rojo-Martinez G, Esteva I, Ruiz-de-Adana MS, Tinahones F, Gomez-Zumaquero JM, Soriguer F: Association between Mspl polymorphism of the APO Al gene and Type 2 diabetes mellitus. Diabet Med 2005, 22(6):782-788

23. Phillips CM, Goumidi L, Bertrais S, Field MR, McManus R, Hercberg S, Lairon D, Planells R, Roche HM: Gene-nutrient interactions and gender may modulate the association between $A p o A 1$ and $A p o B$ gene polymorphisms and metabolic syndrome risk. Atherosclerosis 2011, 214(2):408-414.

24. Reguero JR, Cubero Gl, Batalla A, Alvarez V, Hevia S, Cortina A, Coto E: Apolipoprotein A1 gene polymorphisms and risk of early coronary disease. Cardiology 1998, 90(3):231-235.

25. Wang XL, Liu SX, McCredie RM, Wilcken DE: Polymorphisms at the 5 '-end of the apolipoprotein $\mathrm{Al}$ gene and severity of coronary artery disease. J Clin Invest 1996, 98(2):372-377.

26. Petersen KF, Dufour S, Hariri A, Nelson-Williams C, Foo JN, Zhang XM, Dziura J, Lifton RP, Shulman Gl: Apolipoprotein C3 gene variants in nonalcoholic fatty liver disease. N Engl J Med 2010, 362(12):1082-1089.

27. Kao JT, Wen HC, Chien KL, Hsu HC, Lin SW: A novel genetic variant in the apolipoprotein A5 gene is associated with hypertriglyceridemia. Hum Mol Genet 2003, 12(19):2533-2539.

28. Pennacchio LA, Olivier M, Hubacek JA, Cohen JC, Cox DR, Fruchart JC, Krauss RM, Rubin EM: An apolipoprotein influencing triglycerides in humans and mice revealed by comparative sequencing. Science 2001, 294(5540):169-173.

29. Hsu M, Chang C, Lee K, Sun H, Tsai Y, Kuo P, Young K, Wu C: Central obesity in males affected by a dyslipidemia-associated genetic polymorphism on APOA1/C3/A4/A5 gene cluster. Nutrition and Diabetes 2013. In press.
30. Hsiao WC, Young KC, Lin SL, Lin PW: Estrogen receptor-alpha polymorphism in a Taiwanese clinical breast cancer population: a casecontrol study. Breast Cancer Res 2004, 6(3):R180-R186.

31. Hsiao WC, Cho WC, Lin PW, Lin SL, Lee WY, Young KC: Quantitative profile of estrogen receptor variants/isoforms in Taiwanese women with breast cancer. Eur J Surg Oncol 2006, 32(5):492-497.

32. Barrett JC, Fry B, Maller J, Daly MJ: Haploview: analysis and visualization of LD and haplotype maps. Bioinformatics 2005, 21(2):263-265.

33. Chlebowski RT, Anderson GL, Geller M, Col N: Coronary heart disease and stroke with aromatase inhibitor, tamoxifen, and menopausal hormone therapy use. Clin Breast Cancer 2006, 6(Suppl 2):S58-S64.

34. Hamrita B, Ben Nasr H, Gabbouj S, Bouaouina N, Chouchane L, Chahed K: Apolipoprotein A1-75 G/A and +83 C/T polymorphisms: susceptibility and prognostic implications in breast cancer. Mol Biol Rep 2011, 38(3):1637-1643.

35. Boyd NF, McGuire $V$ : Evidence of association between plasma highdensity lipoprotein cholesterol and risk factors for breast cancer. J Natl Cancer Inst 1990, 82(6):460-468.

36. D'Alessandro A, Scaloni A, Zolla L: Human milk proteins: an interactomics and updated functional overview. J Proteome Res 2010, 9(7):3339-3373.

37. Hodgkinson VC, Agarwal V, EL D, Fox JN, McManus PL, Mahapatra TK, Kneeshaw PJ, Drew PJ, Lind MJ, Cawkwell L: Pilot and feasibility study: comparative proteomic analysis by 2-DE MALDI TOF/TOF MS reveals 143-3 proteins as putative biomarkers of response to neoadjuvant chemotherapy in ER-positive breast cancer. J Proteomics 2012, 75(9):2745-2752

38. Cortesi L, Barchetti A, De Matteis E, Rossi E, Della Casa L, Marcheselli L, Tazzioli G, Lazzaretti MG, Ficarra G, Federico M, et al: Identification of protein clusters predictive of response to chemotherapy in breast cancer patients. J Proteome Res 2009, 8(11):4916-4933.

39. Lee HJ, Paul S, Atalla N, Thomas PE, Lin X, Yang I, Buckley B, Lu G, Zheng X, Lou YR, et al: Gemini vitamin D analogues inhibit estrogen receptorpositive and estrogen receptor-negative mammary tumorigenesis without hypercalcemic toxicity. Cancer Prev Res (Phila) 2008, 1(6):476-484

40. Fitzgerald ML, Mujawar Z, Tamehiro N: ABC transporters, atherosclerosis and inflammation. Atherosclerosis 2010, 211(2):361-370

41. Dean $M: A B C$ transporters, drug resistance, and cancer stem cells. $J$ Mammary Gland Biol Neoplasia 2009, 14(1):3-9.

42. Schimanski S, Wild PJ, Treeck O, Horn F, Sigruener A, Rudolph C, Blaszyk H, Klinkhammer-Schalke M, Ortmann O, Hartmann A, et al: Expression of the lipid transporters $A B C A 3$ and $A B C A 1$ is diminished in human breast cancer tissue. Horm Metab Res 2010, 42(2):102-109.

43. El Roz A, Bard JM, Huvelin JM, Nazih H: LXR agonists and ABCG1dependent cholesterol efflux in MCF-7 breast cancer cells: relation to proliferation and apoptosis. Anticancer Res 2012, 32(7):3007-3013.

44. Bourguignon LY, Gilad E, Rothman K, Peyrollier K: Hyaluronan-CD44 interaction with IQGAP1 promotes Cdc42 and ERK signaling, leading to actin binding, Elk-1/estrogen receptor transcriptional activation, and ovarian cancer progression. J Biol Chem 2005, 280(12):11961-11972.

45. Holm C, Rayala S, Jirstrom K, Stal O, Kumar R, Landberg G: Association between Pak1 expression and subcellular localization and tamoxifen resistance in breast cancer patients. J Natl Cancer Inst 2006, 98(10):671-680.

46. Su F, Kozak KR, Imaizumi S, Gao F, Amneus MW, Grijalva V, Ng C, Wagner A, Hough G, Farias-Eisner G, et al: Apolipoprotein A-I (apoA-I) and apoA-I mimetic peptides inhibit tumor development in a mouse model of ovarian cancer. Proc Natl Acad Sci U S A 2010, 107(46):19997-20002.

47. Su F, Grijalva V, Navab K, Ganapathy E, Meriwether D, Imaizumi S, Navab M, Fogelman AM, Reddy ST, Farias-Eisner R: HDL mimetics inhibit tumor development in both induced and spontaneous mouse models of colon cancer. Mol Cancer Ther 2012, 11(6):1311-1319.

48. Kwan ML, Habel LA, Flick ED, Quesenberry CP, Caan B: Post-diagnosis statin use and breast cancer recurrence in a prospective cohort study of early stage breast cancer survivors. Breast Cancer Res Treat 2008, 109(3):573-579.

doi:10.1186/1471-2407-13-330

Cite this article as: Hsu et al:: The dyslipidemia-associated SNP on the APOA1/C3/A5 gene cluster predicts post-surgery poor outcome in Taiwanese breast cancer patients: a 10-year follow-up study. BMC Cancer 2013 13:330. 